\title{
Time for Clinic: Fourth-Year Primary Care Exposure and Clinic Preparedness Among Internal Medicine Interns
}

\author{
Julia Nath, $M D^{7}{ }^{1}$, Julie Oyler, $M D^{7}$, Amber Bird, $M D^{2}$, Maryann K. Overland, $\mathrm{MD}^{3}$, \\ Lesley King, MD ${ }^{3}$, Christopher J. Wong, MD ${ }^{3}$, Amy W. Shaheen, MD, MSC ${ }^{4}$, and \\ Amber T. Pincavage, $M D^{5}$
}

${ }^{1}$ Internal Medicine Residency Program, University of Chicago Medicine, Chicago, IL, USA; ${ }^{2}$ University of Pennsylvania, Perelman School of Medicine, Philadelphia, PA, USA; ${ }^{3}$ Division of General Internal Medicine, Department of Medicine, University of Washington, Seattle, WA, USA; ${ }^{4}$ University of North Carolina, Chapel Hill, NC, USA; ${ }^{5}$ Pritzker School of Medicine \& Department of Medicine, University of Chicago, Chicago, IL, USA.

BACKGROUND: Internal medicine (IM) residency graduates consistently report being less prepared for outpatient practice than inpatient medicine. Although an initial study suggested interns arriving for IM residency reported low levels of preparedness for continuity clinic, the impact of education and experience during the undergraduate medical education to graduate medical education transition on ambulatory training is unclear.

OBJECTIVE: To describe end of medical school primary care exposure among entering IM interns and its association with self-assessed preparedness for residency continuity clinic.

DESIGN: Cross-sectional survey of 161 entering IM interns in 2019.

PARTICIPANTS: Entering interns at four geographically diverse IM residency programs (University of Chicago, University of North Carolina, University of Pennsylvania, and University of Washington), representing 81 US medical schools.

RESULTS: A total of 139 interns (86\%) responded to the survey. Surveyed interns reported a median of zero days of general internal medicine (GIM) clinic (interquartile range [IQR]: 0-20 days) and 2.5 days of multispecialty adult primary care (IQR: 0-26.5 days) during fourth year of medical school. The median last exposure to primary care was 13 months prior to internship (IQR: 7-18 months). Interns who rated themselves as prepared for primary care clinic reported a median of twenty more multispecialty adult primary care days (20 vs. 0 days; $p<0.01$ ) and fourteen more GIM clinic days ( 14 vs. 0 days; $p<0.01)$ than their unprepared counterparts. The experiences were also more recent, with six fewer months between their last multispecialty adult primary care exposure and the start of internship (9 vs. 15 months; $p<0.01)$.

CONCLUSIONS: The majority of incoming IM interns had no primary care training during the fourth year of medical school. At the start of residency, IM interns who felt more prepared for their primary care clinic reported more recent and more numerous primary care experiences.

Prior Presentations SGIM National Meeting May 6-9, 2020 (conference cancelled), AAIM Academic Internal Medicine Week, April 20, 2020 (conference cancelled).

Received August 3, 2020

Accepted December 22, 2020

Published online February 5, 2021
KEY WORDS: fourth-year medical school; primary care; intern year; resident continuity clinic; medical education.

J Gen Intern Med 36(10):2929-34

DOI: $10.1007 / \mathrm{s} 11606-020-06562-y$

(C) Society of General Internal Medicine 2021

\section{INTRODUCTION}

In recent decades, the USA's volume of inpatient care has decreased markedly, ${ }^{1}$ diverting more care to ambulatory settings. Despite the resultant growing need, there is a projected shortage of primary care providers. ${ }^{2}$ Educators have advocated for enhanced primary care training to address this shortage and improve preparedness of graduates to practice primary care. The plurality of current primary care providers (PCPs) in the USA are general internists, ${ }^{3}$ and internal medicine (IM) residency primary care curricula have expanded to prepare graduates for this role. Additionally, to improve ambulatory training, the Accreditation Council for Graduate Medical Education (ACGME) requires that one-third of IM residency clinical experiences occur in the outpatient setting, and that all residents have at least 130 half-days of a continuity clinic in which the resident is the primary care provider for a panel of patients. ${ }^{4}$ The impact of undergraduate medical education (UME) on the transition into residency continuity clinic and resident comfort with outpatient general internal medicine (GIM) is unclear.

Medical school curricula have also evolved to enhance primary care training in part to meet this need, both globally and within GIM. The Liaison Committee on Medical Education (LCME) now explicitly requires outpatient clinical curricula, though not specifically primary care. ${ }^{5}$ The proportion of medical schools that incorporate GIM ambulatory time into their core (third year) rotations has increased from $85 \%$ in 2013 to $99 \%$ in $2017 .{ }^{6,7}$ Notably, $48 \%$ of that GIM experience occurred in interdepartmental primary care rotations with other specialties such as family medicine and pediatrics. ${ }^{6}$ In 2017, clerkship directors reported that time in GIM clinic ranged from 15 half days in the traditional block model to 31 half days as part of longitudinal integrated clerkships. ${ }^{8}$ 
Despite increased attention to outpatient training in medical school, one IM residency program reported that only $15 \%$ of interns from a diversity of medical schools felt they received sufficient training in medical school to prepare them for their continuity clinic. ${ }^{9}$ One possible reason for the discrepancy between increased medical school primary care curricula and discomfort with primary care among entering IM residents may be that existing training may be insufficient. It may also be due to timing, specifically, long lapses in primary care training experiences prior to the start of residency. Almost all of the increases in primary care time during medical school occurred during the third-year core rotations. ${ }^{7,8}$ In the 1950 s, many medical schools devoted the entire fourth year to ambulatory experiences, but since the 1960s medical schools have reserved most of the fourth year for electives. ${ }^{10}$ The current fourth-year structure includes an inpatient sub-internship but little other curricular standardization to prepare students for their residency. ${ }^{8,11}$ Fewer than one-third of schools require a primary care rotation in the fourth year. ${ }^{8,11}$ Students may participate in elective primary care experiences but there remains a paucity of data describing where and how students actually participate in primary care rotations prior to starting residency. More data about primary care preparedness after medical school and experience prior to the UME to GME transition are needed.

Even after IM residency, graduates report feeling less prepared to manage common outpatient complaints than common inpatient presentations. ${ }^{12}$ This lack of preparedness has been implicated as a significant reason why only $22 \%$ of IM graduates plan a career in primary care on graduation. ${ }^{13}$ It is unclear whether this discomfort with primary care complaints and disinterest in primary care careers among IM residents begins in medical school and perpetuates during residency or where the gap originates. Therefore, understanding where gaps lie at the medical student level in primary care preparedness will be important to enhance primary care training in GME and beyond.

In this multi-site study, we sought to describe primary care exposure prior to residency among a large cohort of IM interns. We then assessed whether the degree of exposure or timing of exposure was associated with self-assessed preparedness for their residency continuity clinic.

\section{METHODS}

\section{Survey}

We created an anonymous sixteen-item survey modeled on prior published instruments that were previously piloted at a single institution with IM interns. ${ }^{8}$ Survey questions were written and revised by authors incorporating best practices of survey design. The authors included subject matter experts with experience in both UME and GME IM outpatient education at multiple institutions as well as an IM resident who represented the intended survey population. It queried demographics (age and gender) and whether the intern was interested in primary care as a career at the time of the survey.

To assess multiple types of primary care exposure during medical school, we asked about rotations in two categories. First, we asked specifically about "Internal Medicine outpatient primary care clinic rotations" (labeled as "GIM" in this study). We then asked about experience in the broader category of "adult primary care clinic rotations (including family medicine, internal medicine, medicine/pediatrics, urgent care)" (labeled as "multispecialty adult primary care" in this study). We chose to query these two categories separately because we were both interested in assessing exposure to GIM specifically but also wanted to capture the fact that many medical school primary care rotations occur across multiple specialties that lead to relevant experience for residency continuity clinic. We asked how many total days of GIM and multispecialty adult primary care clinic rotations interns had during their fourth year of medical school. We then asked for the month and year of their last outpatient GIM rotation and multispecialty adult primary care rotation. (See Appendix A for full survey.)

The survey assessed the interns' overall confidence in managing patients independently in a primary care clinic based on their medical school training using a Likert scale (from 1, not at all confident, to 5 , extremely confident). It further queried the intern's confidence in agenda setting and independently evaluating and managing common primary care conditions (hypertension, diabetes, health maintenance, musculoskeletal complaints, and depression) based on their medical school training. These conditions were selected based upon previous literature on IM resident preparedness for primary care and the authors' consensus regarding these as high yield primary care conditions that should be taught in medical school. ${ }^{9,12}$ Lastly, interns were asked to selfassess their overall preparedness to manage patients independently in a primary care clinic using a Likert scale (from 1 , not at all prepared, to 5 , extremely prepared).

\section{Participants/Setting}

In June through August 2019, we administered the survey to 161 categorical IM and medicine-pediatrics interns at four geographically diverse IM residency programs (University of Chicago, University of North Carolina, University of Pennsylvania, and University of Washington). These interns represented 81 US medical schools. Among the respondents, two international medical graduates were excluded given the aim of our study to examine US medical education. Categorical interns included primary care track and non-primary care track interns.

The survey was administered to interns at the beginning of their first ambulatory rotation, so information was captured prior to any exposure to their residency continuity clinic. As most programs surveyed participate in a block $(\mathrm{x}+\mathrm{y})$ system, the dates of June-August reflect the time necessary for all cohorts to rotate through their first ambulatory rotation block. 


\section{Data Analysis}

We used descriptive statistics to characterize the demographics, primary care exposure, and self-assessed preparedness among interns.

On the five-point Likert scale for overall preparedness, $89 \%$ of interns answered either 2 (slightly prepared) or 3 (moderately prepared). Given this clustering, we dichotomized the variable into prepared $(\geq 3)$ vs. unprepared $(\leq 2)$ for the responsibilities of resident continuity clinic to ease interpretation. While five-point Likert scales are often treated as continuous variables, ${ }^{14}$ it is often recommended to avoid this and use non-parametric methods to analyze the results. We felt it reasonable to lose statistical power to break the variable into a binary and improve interpretation. Additionally, there was consensus among the authors based on experience and previous literature that the difference between slightly prepared and not at all prepared was not likely clinically meaningful and that slightly prepared would be most appropriately categorized as unprepared and moderately prepared would be most appropriately categorized as prepared. ${ }^{12}$

The distribution of days of primary care experience during the fourth year of medical school and months since last primary care experience (GIM or multispecialty adult primary care) were non-normal. Therefore, results are presented as medians with IQR and the statistical significance of comparisons between groups was determined using Wilcoxon ranksum tests. Data analysis was completed using Stata Version 14. The study was determined to be exempt by the Offices of Human Subjects Research at the University of North Carolina, University of Chicago, University of Pennsylvania, and University of Washington.

\section{RESULTS}

A total of 139 interns (86\%) responded to the survey. After excluding two international medical graduates, the final 137 intern sample was $50 \%$ female and averaged 28 years old. They were from 81 regionally diverse medical schools (Table 1). Thirteen percent were interested in primary care as a career. The median last exposure to multispecialty adult primary care was 13 months prior to the start of residency (interquartile range [IQR]: 7-18 months). For GIM clinic specifically, the median last exposure was 16 months prior to the start of residency (IQR: 8-20 months). During the fourth year of medical school, surveyed interns reported a median of zero days of GIM clinic (IQR: 0-20 days) and 2.5 days of multispecialty adult primary care (IQR: 0-26.5 days).

Using the cutoff of 3 (moderately prepared) or greater to define prepared as described in the methods, $54 \%$ of interns felt "unprepared" and $46 \%$ felt "prepared" to manage patients independently in primary care clinic. The interns felt least confident in managing musculoskeletal complaints (average 2.1 ), and most confident in managing hypertension (average 2.9) out of the queried common primary care conditions
(Appendix B). However, there were no major statistical differences in their confidence among these conditions.

Interns who rated themselves as prepared for primary care clinic had more recent experiences in primary care with a median of six fewer months between their last multispecialty adult primary care exposure and the start of internship than those who rated themselves as unprepared (9 vs. 15 months; $p<0.01$; Fig. 1; Table 2). Notably, $68 \%$ of the 63 interns who assessed themselves as prepared for clinic reported having had any adult primary care experience during their fourth year, compared to only $35 \%$ of the 74 interns who rated themselves as unprepared $(p<0.01)$. For GIM specifically, prepared interns reported seven fewer months since their last GIM primary care experience than unprepared interns (11 vs. 18 months; $p<0.01$; Fig. 1; Table 2).

Prepared interns reported a median of twenty more multispecialty adult primary care days ( 20 vs. 0 days; $p<0.01)$ and fourteen more GIM clinic days (14 vs. 0 days; $p<0.01$ ) during the fourth year compared to their counterparts who felt unprepared (Table 2; Fig. 2).

Interns who reported interest in a primary care career reported a median of twenty more multispecialty adult primary care days during their fourth year than those who did not (20 vs. 0 days; $p=0.04$ ). They also reported ten more GIM clinic days, but this did not reach statistical significance (10 vs. 0 days; $p=0.10$ ). There was no significant difference in months since last primary care experience (GIM or multispecialty) or self-reported preparedness for clinic between those indicating they were interested in primary care careers and those who did not.

\section{DISCUSSION}

Our survey of interns across four geographically diverse IM residency programs representing 81 US medical schools indicates that little to no primary care training occurs during the fourth year of medical school. We demonstrate that this lack of training is associated with IM interns reporting lower

Table 1 Demographics of surveyed interns $(n=137)$

\begin{tabular}{ll}
\hline \hline & Mean (range) or $\boldsymbol{n}(\boldsymbol{\%})$ \\
\hline Age & $27.9(24-35)$ \\
Female & $69(50 \%)$ \\
Med school location & \\
Northeast & $32(23 \%)$ \\
Midwest & $45(33 \%)$ \\
South & $28(21 \%)$ \\
West & $30(22 \%)$ \\
Categorical & $133(97 \%)$ \\
Medicine-pediatrics & $4(3 \%)$ \\
Residency site & $31(23 \%)$ \\
University of Chicago & $50(36 \%)$ \\
University of Washington & $31(23 \%)$ \\
University of North Carolina & $25(18 \%)$ \\
University of Pennsylvania & \\
Interested in a primary care career & $48(35 \%)$ \\
No & $72(53 \%)$ \\
Maybe & $17(13 \%)$ \\
Yes &
\end{tabular}




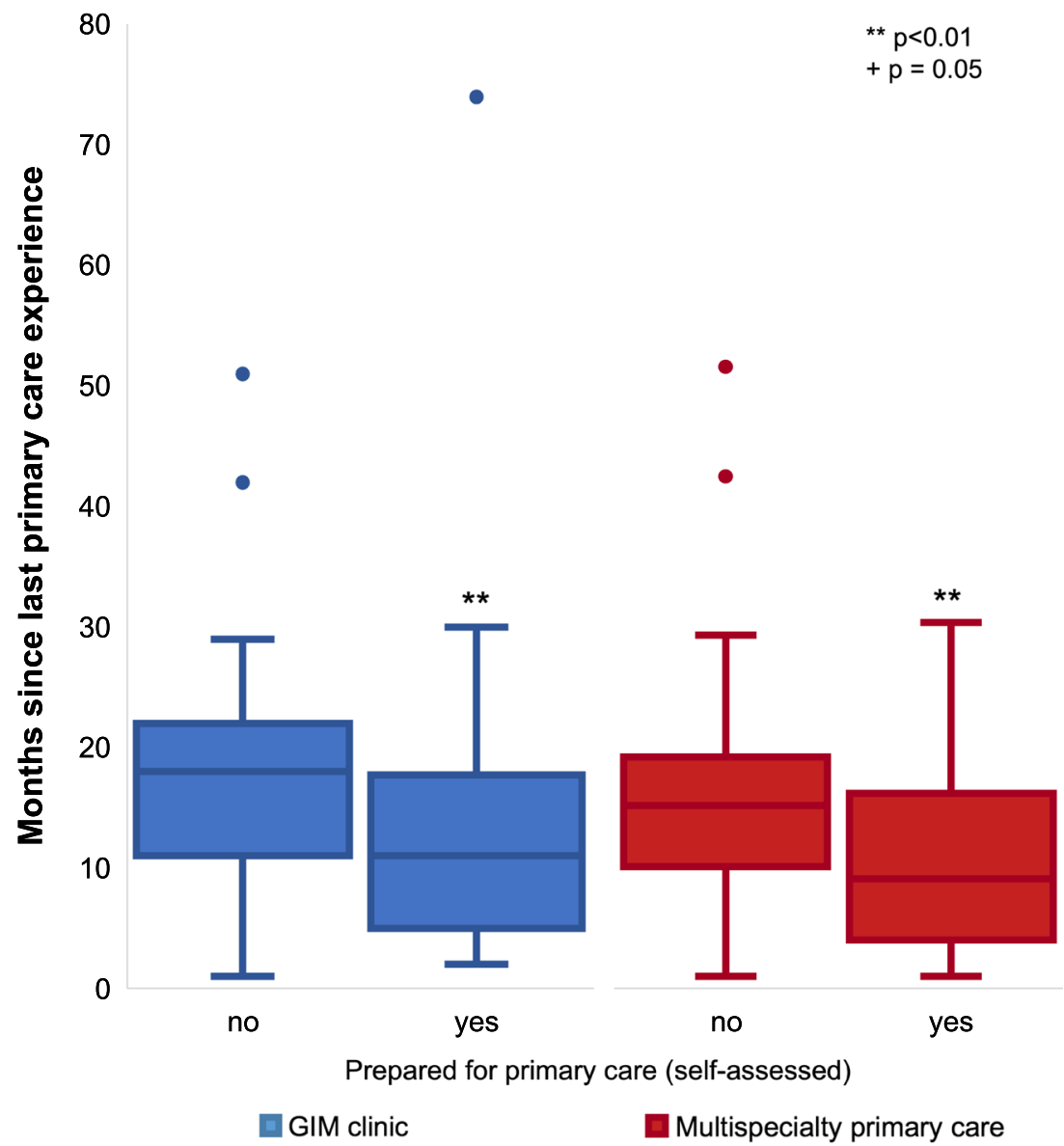

Figure 1 Months since last primary care experience, by self-assessed preparedness for resident continuity clinic. Interns who assessed themselves as unprepared for clinic reported a median of 18 months since their last GIM (general internal medicine) clinic experience (IQR: 11-22 months) and 15 months since their last adult multispecialty (GIM, family medicine, medicine/pediatrics, and other) primary care experience (IQR: 10-19 months). Interns who assessed themselves as prepared for clinic reported a median of 11 months since their last GIM clinic (IQR: 5-17.5 months) and 9 months since their last multispecialty adult primary care experience (IQR: 4-16 months). Both comparisons by Wilcoxon rank-sum tests were statistically significant $(p<0.01)$.

preparedness to manage primary care patients in their continuity clinics.

The fourth year of medical school is a unique opportunity for individualized curricula to suit the student's interests and needs. ${ }^{10,15}$ In order of proportion of students endorsing them, their primary goals for the fourth year are to prepare for residency, to gain admission to their residency of choice, and to broaden their educational experience. ${ }^{16}$ To facilitate these diverse goals, medical schools make few requirements of students, instead offering a breadth of experiences from which to choose. ${ }^{11}$ Presumably, those choices include outpatient-based primary care rotations at many institutions. However, we find that most of the interns we surveyed did not choose to participate in those opportunities despite entering IM, which involves an ACGMEmandated primary care continuity clinic as part of residency. Medical schools could consider encouraging IM-bound fourthyear students to participate in a GIM primary care rotation to help prepare them for their residency continuity clinic.

Notably, there was a low rate of expressed interest in primary care careers among our sample (13\%) although this was similar to rates reported in previous studies $(14.9 \%){ }^{13}$
Although those interested in primary care did report more clinic days during their fourth year, they did not report more recent experiences. This finding may indicate that those with an interest in primary care are seeking out more primary care

Table 2 Fourth-year primary care experience by self-assessed preparedness for residency continuity clinic

\begin{tabular}{llll}
\hline \hline & Unprepared & Prepared & $p$ value \\
\hline $\begin{array}{l}\text { Median days of fourth-year } \\
\text { clinic (IQR) }\end{array}$ & & & \\
$\quad$ GIM & $0(0-14)$ & $14(0-20)$ & $p<0.01$ \\
$\quad$ Multispecialty adult & $0(0-14)$ & $20(0-36)$ & $p<0.01$ \\
primary care & & & \\
$\begin{array}{l}\text { Median months since last } \\
\text { experience (IQR) }\end{array}$ & $18(11-22)$ & $11(5-17.5)$ & $p<0.01$ \\
$\quad$ GIM & $15(10-19)$ & $9(4-16)$ & $p<0.01$ \\
$\begin{array}{l}\text { Multispecialty adult } \\
\text { primary care }\end{array}$ & & & \\
\hline
\end{tabular}

In our survey, general internal medicine (GIM) clinics is defined as "Internal Medicine outpatient primary care clinic rotations"; multispecialty adult primary care was defined as "adult primary care clinic rotations (including family medicine, internal medicine, medicinel pediatrics, urgent care)." IQR, interquartile range 


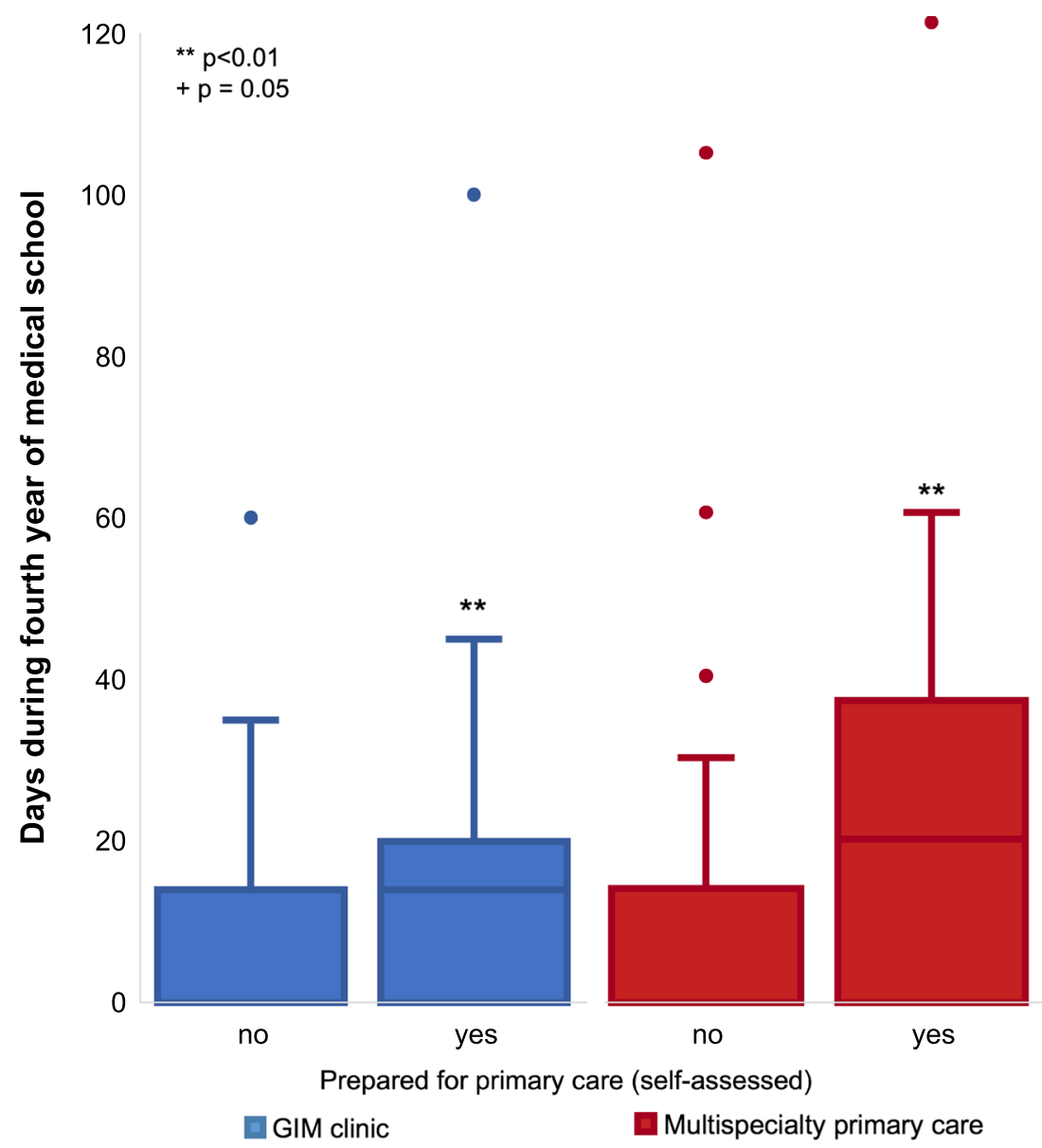

Figure 2 Days of primary care clinic during fourth year of medical school, by self-assessed preparedness for resident continuity clinic. Interns who assessed themselves as unprepared for clinic reported a median of 0 days of GIM (general internal medicine) clinic (IQR: 0-14 days) and 0 days of multispecialty (GIM, family medicine, medicine/pediatrics, and other) adult primary care clinic (IQR: 0-14 days). Interns who assessed themselves as prepared for clinic reported a median of 14 days of GIM clinic (IQR: 0-20 days) and 20 days of multispecialty adult primary care (IQR: 0-36 days) during their fourth year. The difference in days of both multispecialty and GIM primary care experience during fourth year between unprepared and prepared interns was statistically significant $(p<0.01)$.

experiences during their fourth year, or that those with those experiences develop an interest in primary care. Further research is needed to understand the motivations of fourth-year students bound for IM to pursue or forgo primary care experiences given the student-driven nature of the year.

While we find that those interns who had primary care experience during their fourth year felt more prepared for their residency clinics, this does not necessarily indicate that requiring fourth-year primary care experiences would improve their comfort in clinic. However, we hope these results will prompt additional studies of adding primary care experiences into fourth-year curricula and the effects on preparedness for residency continuity clinic. Several previous reviews have provided frameworks for primary care curricula,${ }^{17}$ which could easily extend into the fourth year in various forms. No one standard fourth-year primary care experience has been described or prescribed by governing bodies, and this is therefore an interesting area for curricular development and evaluation. However, even if additional curriculum in the fourth year were evaluated and shown to improve intern readiness (selfreported or otherwise) for clinic, additional barriers likely still exist to students participating in additional clinic time including clinic space, availability of preceptors, time barriers, and finances. ${ }^{7,8,18}$

This demonstration of the lack of preparation and selfassessed low levels of preparedness among incoming interns may indicate a need for remediation and supervision during residency. Introductory "boot camps" have been used either late in the fourth year or early in residency to help bridge some of the gaps in this transition, at least one of which had a primary care focus. ${ }^{9}$ In addition, graduated levels of supervision are likely necessary in continuity clinics. Preceptors may need to take a very active role in early intern visits and provide assistance with issues of follow-up and results outside of clinic.

Our study has several limitations. It is cross-sectional and thus no inference of causation can be drawn from our results. We relied on self-report of retrospective data that is subject to recall bias. For example, students who were at medical schools with a stronger focus on primary care curricula might both remember their primary care experiences more clearly and report more accurately. It is unknown whether self-reported 
preparedness for clinic translates into competency-based clinic readiness. Given calls for better aligning assessment and performance metrics in UME and GME, additional research will be helpful in determining if self-assessed preparedness correlates with metrics of objective performance in continuity clinic and if fourth-year primary care experiences translate into objective improvements in intern clinic preparedness and performance. ${ }^{19}$ In addition, our multispecialty adult primary care category, which was intended to inquire about adult primary care rotations, could have been interpreted by the intern to include specialty experiences which may be less salient to primary care clinic preparedness. Finally, while our sample is geographically diverse, it is not necessarily representative of the national IM intern population.

It is notable that we capture data at a time of intense transition when starting residency, before starting clinic. While surveying interns at a point later in the year might capture a more complete picture of preparedness for clinic, it would also be confounded by residency experience. Surveying interns at this time captures their perceptions of their preparedness based on their medical school experiences alone and is closer to that experience to better quantify it.

To our knowledge, this is the first survey to assess IM intern exposure to primary care prior to residency and demonstrates a gap in the current curricular structure. Further, we demonstrate that absence of this training is associated with IM interns feeling less prepared for their continuity clinic. Given that this feeling of ill preparedness for primary care persists through graduation, ${ }^{12}$ this study highlights an important opportunity to trial curricular innovations in the fourth year or intern year to bridge this gap.

In conclusion, we find that during their fourth year of medical school, the median incoming IM intern had zero days of GIM primary care clinic and only 2.5 days of adult primary care rotations in any specialty. At the start of residency, IM interns who felt more prepared for their primary care clinic reported more recent and more numerous primary care experiences. Including more primary care experiences during fourth-year training could improve preparedness of medical students who are pursuing IM. Additionally, enhancing initial levels of intern supervision in continuity clinic or increasing intern primary care training experiences may be helpful.

Corresponding Author: Amber T. Pincavage, MD; Pritzker School of Medicine \& Department of Medicine, University of Chicago, Chicago, IL, USA (e-mail: apincava@medicine.bsd.uchicago.edu).
Supplementary Information The online version contains supplementary material available at https://doi.org/10.1007/s11606-02006562-y

\section{REFERENCES}

1. McDermott KW, Elixhauser A, Sun R. Trends in hospital inpatient stays in the United States, 2005-2014. HCUP Statistical Brief 2017; https:// www.hcup-us.ahrq.gov/reports/statbriefs/sb_hospoverview.jsp.

2. Health Resources and Services Administration Bureau of Health Professions. Projecting the Supply and Demand for Primary Care Practitioners Through 2020. 2013; https://bhw.hrsa.gov/health-workforce-analysis / primary-care-2020.

3. Kaiser Family Foundation. Professionally Active Primary Care Physicians by Field. 2019; https://www.kff.org/other/state-indicator/primary-carephysicians-by-field.

4. ACGME Program Requirements for Graduate Medical Education in Internal Medicine. 2019; https://www.acgme.org/Portals/0/PFAssets/ ProgramRequirements/140_InternalMedicine_2019.pdf.

5. Liason Committee on Medical Education. Functions and Structure of a Medical School: Standards for Accreditation of Medical Education Programs Leading to the MD Degree. Washington, DC: Association of American Medical Colleges and American Medical Association; 2020.

6. Shaheen AW, Alexandraki I, Fazio SB, et al. The State of Ambulatory Undergraduate Internal Medicine Medical Education: Results of the 2016 Clerkship Directors in Internal Medicine Annual Survey. Am J Med 2019;132(5):652-657.

7. Shaheen A, Papp KK, Torre D. The internal medicine clerkship and ambulatory learning experiences: results of the 2010 clerkship directors in internal medicine survey. Teach Learn Med 2013;25(3):225-230.

8. Pincavage AT, Fagan MJ, Osman NY, et al. A National Survey of Undergraduate Clinical Education in Internal Medicine. J Gen Intern Med 2019;34(5):699-704.

9. Esch LM, Bird AN, Oyler JL, Lee WW, Shah SD, Pincavage AT. Preparing for the primary care clinic: an ambulatory boot camp for internal medicine interns. Med Educ Online 2015;20:29702.

10. Whitcomb ME. Ambulatory-based clinical education: Flexner revisited. Acad Med 2006;81(2):105-106.

11. Elnicki DM, Gallagher S, Willett L, et al. Course Offerings in the Fourth Year of Medical School: How U.S. Medical Schools Are Preparing Students for Internship. Acad Med 2015;90(10):1324-1330.

12. Wiest FC, Ferris TG, Gokhale M, Campbell EG, Weissman JS, Blumenthal D. Preparedness of internal medicine and family practice residents for treating common conditions. JAMA. 2002;288(20):2609-2614.

13. West CP, Dupras DM. General Medicine vs Subspecialty Career Plans Among Internal Medicine Residents. JAMA. 2012;308(21):2241-2247.

14. Jamieson S. Likert scales: How to (ab) use them? Med Educ 2004;38(12):1217-1218.

15. Sklar DP. Making the fourth year more meaningful. Acad Med 2014;89(4):527-528.

16. Benson NM, Stickle TR, Raszka WV, Jr. Going "Fourth" From Medical School: Fourth-Year Medical Students' Perspectives on the Fourth Year of Medical School. Acad Med 2015;90(10):1386-1393.

17. Fazio SB, Demasi M, Farren E, et al. Blueprint for an undergraduate primary care curriculum. Acad Med 2016;91(12):1628-1637.

18. Bowen $\mathbf{J}$, Irby DM. Assessing quality and costs of education in the ambulatory setting: a review of the literature. Acad Med 2002;77(7):621-680.

19. Chen HC, van den Broek WS, ten Cate O. The case for use of entrustable professional activities in undergraduate medical education. Acad Med 2015;90(4):431-436.

Publisher's Note: Springer Nature remains neutral with regard to jurisdictional claims in published maps and institutional affiliations. 